\title{
Alcance en el uso de cámara no midriática para la evaluación de fondo de ojo de sujetos con diabetes
}

\section{Scope of non-mydriatic camera use for fundus evaluation in subjects with diabetes}

\author{
Virgilio Lima-Gómez ${ }^{1}$, M. Guadalupe Colas-Calvere ${ }^{1}$, Selma A. Somilleda-Ventura ${ }^{2}$ \\ y Dulce M. Razo Blanco-Hernández ${ }^{2 *}$
}

${ }^{1}$ Servicio de Oftalmología; '2División de Investigación. Hospital Juárez de México, Ciudad de México, México

\begin{abstract}
Background: Predominantly peripheral lesions (PPLs) could be any sign of diabetic retinopathy that only occurs outside the central $30^{\circ}$ of the retina. These lesions represent an associated progression risk factor of retinopathy. Methods: An observational, cross-sectional, prospective, comparative study in subjects with (Group 1) or without (Group 2) diabetes was conducted. We determined the proportion and 95\% confidence intervals of retinopathy level, the proportion of peripheral retinal changes, and the proportion of eyes with only PPLs of retinopathy. We compared peripheral retinal changes between subjects with and without diabetes by $\chi^{2}$ test. Results: Two hundred subjects were evaluated, $77 \%$ female, mean age of $54.6 \pm 7.35$ years. In Group 1, 27\% had some level of diabetic retinopathy and in $23 \%$, it was bilateral. Only $2 \%$ of these subjects had PPLs. Peripheral retinal changes not associated to diabetes were found in $47 \%$ in Group 1 vs. $42 \%$ in Group 2 ( $p>0.05$ ). Conclusions: The conventional non-mydriatic camera for fundus eye photography is safe to evaluate subjects with diabetes and without ocular symptoms, in which cases peripheral retinal exploration could be omitted.
\end{abstract}

Key words: Detection. Diabetes. Diabetic retinopathy.

\section{RESUMEN}

Antecedentes: Las lesiones predominantemente periféricas pueden ser cualquier signo de la retinopatía diabética que se encuentre únicamente por fuera de los $30^{\circ}$ de la retina central. La presencia de estas es un factor asociado a mayor progresión de la enfermedad. Métodos: Estudio observacional, transversal, prospectivo y comparativo en sujetos con (grupo 1) y sin (grupo 2) diabetes, donde se determinó la proporción e intervalos de confianza del 95\% del grado de retinopatía, la proporción de cambios en la retina periférica y la proporción de ojos con únicamente lesiones predominantemente periféricas por diabetes. Se compararon cambios en la retina periférica entre sujetos sanos y con diabetes mediante prueba de $\chi^{2}$. Resultados: Se evaluaron 200 sujetos, el $77 \%$ fueron de sexo femenino y la edad promedio fue $54.6 \pm 7.35$. En el grupo 1, el 27\% presentó algún grado de retinopatía y en el 23\% esta fue bilateral; solo el $2 \%$ presentó lesiones predominantemente periféricas. Se encontraron cambios en retina periférica no asociados a diabetes en el $47 \%$ de los casos en el grupo 1 vs. un $42 \%$ en el grupo 2 ( $p>0.05$ ). Conclusiones: La fotografía de fondo de ojo con cámara no midriática convencional es segura para evaluar a los sujetos con diabetes, y cuando no exista sintomatología ocular pudiera omitirse la exploración de la retina periférica en estos pacientes.

Palabras clave: Diabetes. Retinopatía diabética. Detección.

\section{Correspondence:}

*Dulce M. Razo Blanco-Hernández

E-mail: razoblanco.dulce@gmail.com
Date of reception: $17-02-2020$

Date of acceptance: $21-01-2021$

DOI: 10.24875/HMCM.20000006
Available online: $25-06-2021$

Hosp Med Clin Manag. 2020;13:143-8

2604-0018 / @ 2021 Mexican Regional Hospitals of High Specialty and Federal Hospitals. Published by Permanyer. This is an open access article under the CC BY-NC-ND license (http://creativecommons.org/licenses/by-nc-nd/4.0/). 


\section{INTRODUCCIÓN}

La retinopatía diabética es una de las complicaciones que se presentan con mayor frecuencia en sujetos con diabetes. Se ha reportado una prevalencia del $28 \%$ en sujetos con conocimiento de la enfermedad y hasta en un $11 \%$ en sujetos recién diagnosticados ${ }^{1}$.

Los microaneurismas son de las primeras manifestaciones clínicas oftalmológicas visibles por medio de la exploración de fondo de ojo; cuando aumentan, pueden originar isquemia retiniana por oclusión de los plexos capilares. Si permanecen permeables, podrían separar a los fotorreceptores al engrosar la retina; cuando se cierran, inducen la formación de exudados por depósito de las lipoproteínas séricas que permanecen a pesar de la reabsorción de líquido a través de los capilares adyacentes ${ }^{2}$. Otras manifestaciones de la enfermedad son la presencia de hemorragias retinianas y manchas algodonosas.

Otros signos de isquemia retiniana que se presentan son la tortuosidad venosa, la dilatación de plexos capilares junto a las zonas de isquemia, conocidas como anormalidades microvasculares intrarretinianas (AMIR) ${ }^{3}$, y la neovascularización que se encuentra en el disco óptico o en cualquier zona de la retina (NVE).

Los microaneurismas/hemorragias retinianas, tortuosidad venosa, AMIR y NVE son lesiones que pueden presentarse en cualquier parte de la retina. Se las denomina lesiones predominantemente en la periferia (predominantly peripheral lesions, PPL) cuando cualquiera de ellas se presenta únicamente en los campos periféricos al estándar de oro. Se ha reportado que las PPL llegan a presentarse hasta en el $21.3 \%$ de los pacientes diabéticos ${ }^{4}$. Las PPL están asociadas con un riesgo de 3.2 veces para la progresión de cualquier grado de la retinopatía y de hasta 4.7 veces más de proliferar en los siguientes cuatro años ${ }^{5}$.

El estándar de oro para la evaluación de la retinopatía diabética es la fotografía retiniana de siete campos del ETDRS (Early Treatment Diabetic Retinopathy Study). Esta evaluación se efectúa mediante la toma de imágenes retinianas de $30^{\circ}$; se obtienen 14 fotos de los siete campos estandarizados que se unen y dan en total una superficie aproximada del $30 \%$ de toda la retina. También se puede hacer el diagnóstico mediante el examen en lámpara de hendidura o con oftalmoscopio indirecto; sin embargo, estos métodos requieren de una midriasis farmacológica para poder realizarlos ${ }^{6}$.

Las nuevas tecnologías pueden realizar las fotografías sin midriasis farmacológica, e incluso hacerlas por medio de un teléfono inteligente (smartphone), con una sensibilidad y especificidad de alrededor del 90\%; estas han comenzado a sustituir al estándar de $\mathrm{oro}^{7}$. Una de las desventajas de estos equipos es que abarcan solo los $45^{\circ}$ centrales, y para poder evaluar la periferia debe tomarse una serie de fotografías y realizar un fotomontaje para alcanzar hasta los $75^{\circ}$ del campo retiniano ${ }^{8}$.

La cámara de fondo de ojo de campo ultraamplio (ultrawide-field imaging) permite una evaluación más sencilla de la fotografía, abarca un campo de hasta $200^{\circ}$, no requiere midriasis y se ha utilizado para la detección de retinopatía diabética, ya que su ángulo es suficiente para evaluar la mayor parte de la retina, incluyendo la periferia9. Por ello desarrollamos un estudio para identificar la proporción de casos en los que se presentaran solo PPL por retinopatía diabética por fuera de los $45^{\circ}$ centrales.

\section{MÉTODOS}

\section{Diseño y población de estudio}

Estudio observacional, transversal, prospectivo y comparativo en sujetos con y sin diabetes de la Ciudad de México y su área metropolitana; la muestra se obtuvo de trabajadores y familiares de un hospital federal de referencia. El estudio se efectuó del 1 de septiembre de 2017 al 31 de marzo de 2018, y recibió la autorización de los comités de investigación y ética del hospital.

Se incluyeron sujetos sin sintomatología ocular en el momento de la evaluación, con diabetes, con y sin retinopatía, de cualquier edad y sexo, que aceptaran participar en el estudio mediante consentimiento informado por escrito. No se incluyeron los sujetos con cirugía intraocular o tratamientos para lesiones retinianas periféricas previos, quienes tuvieran opacidades en el segmento anterior que no permitieran obtener una adecuada imagen del fondo del ojo, y quienes tuvieran mala fijación visual central. Se eliminaron los sujetos con ojos únicos y a quienes retiraron su consentimiento bajo información. 


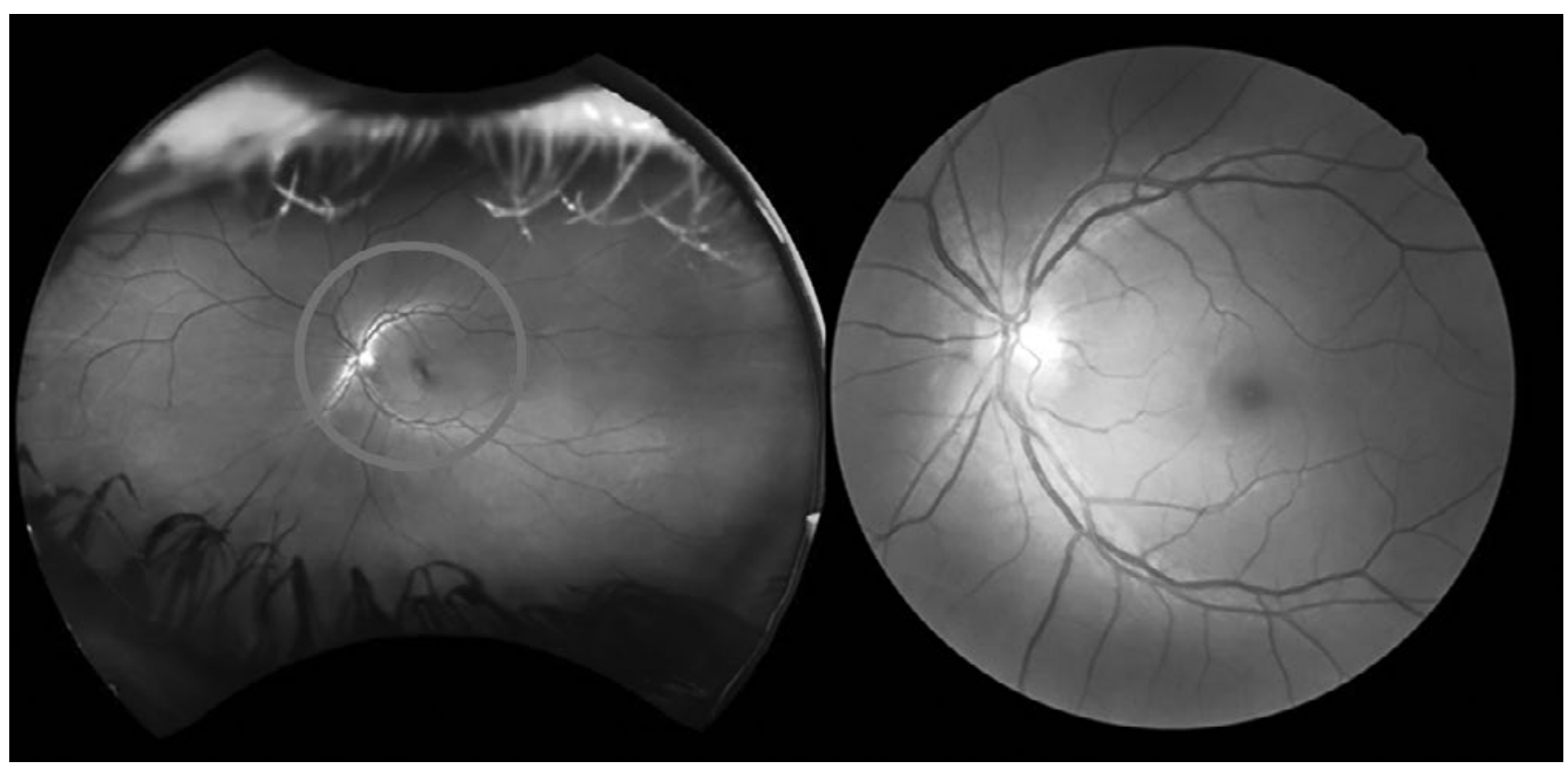

Figura 1. Fotografía de fondo de ojo de paciente mediante dos equipos. A la izquierda, fotografía de fondo de ojo con cámara de campo ultraamplio con el equipo California (Optomap Ultra-Widefield ${ }^{\circledR}$ System by Optos $^{\circledR}$ ). A la derecha, fotografía de fondo de ojo con cámara no midriática convencional. El círculo muestra los $45^{\circ}$ centrales que se toman en las fotografías de fondo de ojo de manera estandarizada, que incluye la visualización del nervio óptico y del área macular.

Las variables de estudio fueron: 1) el grado de retinopatía diabética, definida por la clasificación del ETDRS, y 2) la frecuencia de lesiones predominantemente periféricas de retinopatía diabética, definida como cualquier lesión existente localizada únicamente por fuera de los $45^{\circ}$ centrales. Además, se determinó la presencia de cambios en la retina periférica, que se definió como la presencia de hallazgos en la retina periférica, como blanco sin presión, degeneración microquística, degeneración en encaje, lesiones empedrado, desprendimiento de retina subclínico y agujeros retinianos.

\section{Examen de fondo de ojo}

Un investigador del estudio obtuvo una imagen de campo ultraamplio con el equipo California (Optomap Ultra-Widefield ${ }^{\circledR}$ System by Optos ${ }^{\circledR}$ ) en ambos ojos de cada sujeto, sin dilatar la pupila. La fotografía tomada de fondo de ojo se dividió en dos regiones: la primera región son los $45^{\circ}$ centrales, área que corresponde a una fotografía con cámara de fondo de ojo convencional, y la segunda región es el resto de la imagen (Fig. 1). Un investigador especialista en retina evaluó las fotografías para calificar el grado de retinopatía diabética y determinar si existían otros cambios en la retina periférica, como blanco sin presión, degeneración microquística, degeneración en encaje, lesiones empedrado, desprendimiento de retina subclínico y agujeros retinianos. Además, identificó las lesiones en toda la retina y las lesiones exclusivamente por fuera de los $45^{\circ}$ centrales.

Un investigador adicional realizó un muestreo aleatorizado para seleccionar 100 sujetos con diabetes, que conformaron un grupo de estudio (grupo 1), y se eligieron 100 sujetos sin diabetes de la misma edad y sexo como grupo de referencia (grupo 2).

\section{Análisis estadístico}

Se determinaron las frecuencias e intervalos de confianza del 95\% (IC 95\%) de retinopatía diabética y de cambios en la retina periférica por paciente y por ojo, y se identificó la proporción de casos en que la afección era bilateral. Se identificó la proporción e IC 95\% de ojos con retinopatía diabética en los cuales la enfermedad se localizaba por fuera de los $45^{\circ}$ centrales. Adicionalmente, se compararon la frecuencia de cambios en la retina periférica por grupo y, en el grupo 1 , entre sujetos con y sin retinopatía diabética, mediante $\chi^{2}$. Se consideró significativo un valor de $p<0.05$. La información se almacenó y analizó con el software SPSS para Windows versión 22. 
Tabla 1. Distribución de los grados de retinopatía en los ojos de los sujetos con diabetes

\begin{tabular}{lcc}
\hline & $\mathbf{n ~ ( \% )}$ & IC $\mathbf{9 5 \%}$ \\
\hline Sin retinopatía & $150(75)$ & $69-81$ \\
No proliferativa muy leve & $22(11)$ & $6.7-15.3$ \\
No proliferativa leve & $16(8)$ & $4.2-11.8$ \\
No proliferativa moderada & $6(3)$ & $0.6-5.4$ \\
No proliferativa severa & - & - \\
No proliferativa muy severa & - & - \\
Proliferativa leve-moderada & - & - \\
Proliferativa de alto riesgo & $6(3)$ & $0.6-5.4$ \\
\hline
\end{tabular}

IC 95\%: intervalo de confianza del $95 \%$.

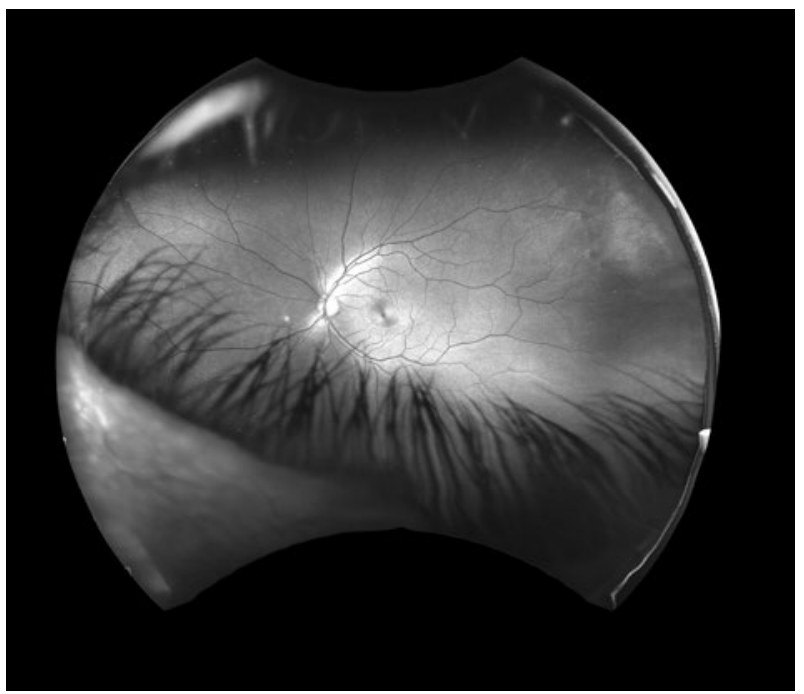

Figura 2. Retinopatía diabética leve. Presencia de microaneurisma por fuera de los $45^{\circ}$ centrales, que con una foto convencional no hubiera sido posible diagnosticarla.

\section{RESULTADOS}

Se evaluaron 200 ojos de 100 pacientes por grupo; 77 pacientes en cada grupo eran sujetos del sexo femenino y la edad promedio fue $54.6 \pm$ desviación estándar (DE) 7.35 años. En los pacientes con diabetes (Grupo 1) el tiempo de evolución fue de 0.1 a 30 años, con promedio de $8.6 \pm 6.49$ años.

En el grupo 1 se encontró retinopatía diabética en 27 sujetos (27\%; IC 95\%: 18.3-38.7); en 23 era bilateral y simétrica (23\%; IC 95\%: 14.8-31.2), y en los cuatro restantes afectaba solo un ojo (bilateralidad $85.2 \%$; IC 95\%: 78.2-92.2). Se encontraron cambios en la retina periférica no asociada a diabetes de 47 sujetos ( $47 \%$; IC 95\%: 37.2-56.8); en 14 eran bilaterales y en 33 unilaterales (bilateralidad 29.78\%; IC 95\%: 16.7-42.9). En el grupo 2 se encontraron cambios en la retina
Tabla 2. Distribución de los cambios retinianos periféricos en cada grupo, por ojo

\begin{tabular}{lccccc}
\hline Cambio periférico & $\begin{array}{c}\text { Grupo 1 } \\
(\mathbf{n = 2 0 0 )}\end{array}$ & \multicolumn{2}{c}{$\begin{array}{c}\text { Grupo 2 } \\
(\mathbf{n}=\mathbf{2 0 0})\end{array}$} & $\mathbf{p}^{*}$ \\
\hline Degeneración microcistoide & 29 & $14.5 \%$ & 42 & $21 \%$ & 0.07 \\
Blanco sin presión & 22 & $11 \%$ & 17 & $8.5 \%$ & 0.4 \\
Degeneración en empedrado & 3 & $1.5 \%$ & 2 & $1.5 \%$ & 1 \\
Encaje & 3 & $1.5 \%$ & 0 & - & 0.1 \\
Desprendimiento de retina & 2 & $1 \%$ & 0 & - & 0.4 \\
subclínico & 59 & $29.5 \%$ & 61 & $30.5 \%$ & 1 \\
Total & & & & & \\
\hline
\end{tabular}

*Prueba de $\chi^{2}$.

Tabla 3. Frecuencia de cambios periféricos retinianos en sujetos con diabetes con y sin retinopatía diabética $\left(\chi^{2}, p=1.0\right)$

\begin{tabular}{lccc}
\hline $\begin{array}{l}\text { Retinopatía } \\
\text { diabética }\end{array}$ & $\begin{array}{c}\text { Con cambios } \\
\text { en la retina } \\
\text { periférica, n }\end{array}$ & $\begin{array}{c}\text { Sin cambios } \\
\text { en la retina } \\
\text { periférica, }\end{array}$ & Total \\
\hline Presente & 13 & 14 & 27 \\
Ausente & 34 & 39 & 73 \\
Total & 47 & 53 & 100 \\
\hline
\end{tabular}

periférica de 42 sujetos (42\%; IC 95\%: 32.2-51.7); en 23, la afectación fue bilateral y en 19, unilateral (bilateralidad 54.76\%; IC 95\%: 39.7-69.8) (Tabla 1).

En dos sujetos del Grupo 1 (2\%, IC 95\% 0-4.7\%) se encontraron PPL del tipo microaneurisma, clasificando a la retinopatía diabética como leve, que no hubieran podido detectarse mediante la evaluación de los $45^{\circ}$ centrales (Fig. 2).

La distribución de los cambios periféricos no asociados a diabetes por ojo en cada grupo se presenta en la tabla 2. En 13 sujetos (13\%; IC 95\%: 6.4-19.6) coexistieron retinopatía diabética y otros cambios en la retina periférica en al menos un ojo. La frecuencia de cambios periféricos fue similar entre los sujetos con y sin retinopatía diabética (Tabla 3).

\section{DISCUSIÓN}

El 2\% de los sujetos evaluados presentaron PPL del tipo de microaneurismas, que pudieran haber clasificado a la retinopatía como de menor grado si se hubieran evaluado con solo una fotografía de $45^{\circ}$. Estos resultados no concuerdan con los publicados previamente en otras poblaciones, en donde hasta el $21 \%$ de los pacientes presentaba alguna PPL, lo que sugería que 
hasta el 9\% de los sujetos evaluados pudiera tener un grado mayor de la retinopatía que no podría detectarse con la evaluación mediante el estándar de oro ${ }^{10}$.

El $47 \%$ de los sujetos diabéticos presentó otros cambios retinianos periféricos, que fueron similares a los cambios encontrados en sujetos sin diabetes $(42 \%)$. Solo en el $13 \%$ de los sujetos coexistieron cambios retinianos periféricos con lesiones por retinopatía diabética.

Se ha reportado que la frecuencia de cambios periféricos con riesgo de desprendimiento de retina en población general puede variar desde el $0.4 \%^{11}$ hasta el $6 \%$. El comportamiento en los sujetos con diabetes fue semejante, puesto que solo el $2 \%$ de nuestros pacientes evaluados requirió tratamiento por estas causas; esto también se ha estudiado en otros países, encontrando una situación similar ${ }^{12}$. Ello podría indicar que la diabetes, e incluso la retinopatía, no son factores que condicionen mayor riesgo para presentar otro tipo de lesiones en la periferia no relacionados con la diabetes.

Aunque el tamaño de muestra no fue amplio, la proporción de pacientes diabéticos que presentó retinopatía diabética fue semejante a la reportada por otros estudios en nuestra población (27 vs. $17.3 \%{ }^{13}$ ) y en poblaciones de otros países ${ }^{14,15}$. A pesar del tiempo y las estrategias utilizadas para el control de la enfermedad, no se ha logrado disminuir la frecuencia de esta complicación; sin embargo, sí se ha reportado una disminución en la frecuencia de casos con lesiones que pongan en riesgo la función visual ${ }^{16,17}$. Evaluar el fondo de ojo del sujeto recién diagnosticado con diabetes es una necesidad primordial para evitar en lo posible la pérdida visual ${ }^{16}$ debido a que, al identificar la gravedad de la retinopatía, se determina la frecuencia del seguimiento y cuándo es apropiado intervenir con un tratamiento. Si se identifican PPL, esto eleva hasta un 4.7\% el riesgo para que la enfermedad progrese en corto tiempo ${ }^{4}$. Por ello, existe la necesidad de continuar con la búsqueda de nuevas estrategias para incidir en el manejo óptimo de los pacientes diabéticos de manera integral y con ello minimizar en lo posible este tipo de complicaciones.

La evaluación de la retina es crucial para el diagnóstico, tratamiento y seguimiento de los sujetos con diabetes y con retinopatía diabética. Sin embargo, la evaluación de la periferia requiere cámaras de campo ultraamplio muy costosas o la exploración bajo midriasis farmacológica, con un tiempo mayor en la consulta, además de las molestias que genera en los pacientes la dilatación pupilar. Los estudios de costo/efectividad de los programas de tamizaje con las cámaras de fondo de ojo sin midriasis han demostrado un beneficio mayor ${ }^{18}$; se omite la molestia de la midriasis pupilar en el paciente y se facilita la evaluación acortando el tiempo de la consulta, y en este estudio encontramos que puede diagnosticar correctamente a más del $95 \%$ de los casos evaluados.

Sin embargo, es importante mencionar que si durante la parte del interrogatorio dirigido en la evaluación inicial se detecta cualquier sintomatología que haga sospechar de lesiones con riesgo de desprendimiento de retina, será necesaria la referencia inmediata con el especialista para una evaluación más completa que permita identificar cualquier tipo lesión.

Una de las fortalezas del estudio fue que se obtuvo una muestra homogénea al parear por edad y sexo y con ello evitar los sesgos que pudieran presentarse por las lesiones que pueden ser más frecuentes en cierta edad o sexo.

Una limitación potencial del estudio fue que solo se tomaron las imágenes de la fotografía de campo ultraamplio y no se comparó con otra fotografía tomada al mismo tiempo con alguna cámara de fondo de ojo sin midriasis. Sin embargo, esta limitación se disminuyó al haber homogenizado la muestra por edad y sexo y haber realizado la estimación de los $45^{\circ}$ centrales para identificar las lesiones que estuvieran por fuera de estos.

\section{CONCLUSIONES}

La fotografía de fondo de ojo con la cámara de fondo de ojo no midriática de tipo convencional $\left(45^{\circ}\right)$ es segura para evaluar a los sujetos con diabetes y, si no hay sintomatología ocular, pudiera omitirse la exploración de la retina periférica en ellos.

\section{FINANCIAMIENTO}

La presente investigación no ha recibido ninguna beca específica de agencias de los sectores público, comercial o sin ánimo de lucro. 


\section{CONFLICTO DE INTERESES}

Ninguno.

\section{RESPONSABILIDADES ÉTICAS}

Protección de personas y animales. Los autores declaran que para esta investigación no se han realizado experimentos en seres humanos ni en animales.

Confidencialidad de los datos. Los autores declaran que han seguido los protocolos de su centro de trabajo sobre la publicación de datos de pacientes.

\section{Derecho a la privacidad y consentimiento informado.} Los autores han obtenido el consentimiento informado de los pacientes y/o sujetos referidos en el artículo. Este documento obra en poder del autor de correspondencia.

\section{BIBLIOGRAFÍA}

1. Pasquel FJ, Hendrick AM, Ryan M, Cason E, Ali MK, Venkat NKM. Costeffectiveness of different diabetic retinopathy screening modalities. J Diabetes Sci Technol. 2016;10(2):301-7.

2. Ciulla TA, Amador AG, Zinman B. Diabetic retinopathy and diabetic macular edema: pathophysiology, screening, and novel therapies. Diabetes Care. 2003;26:2653-64

3. American Academy of Ophthalmology Retina Panel. Preferred Practice Pattern Guideline. Diabetic Retinopathy. San Francisco, CA: American Academy of Ophthalmology; 2008

4. Silva PS, Cavallerano JD, Haddad NMN, Kwak H, Dyer KH, Omar AF, et al. Peripheral lesions identified on ultrawide field imaging predict increased risk of diabetic retinopathy progression over 4 years. Ophthalmology. 2015;122(5):949-56

5. Silva PS, Dela Cruz AJ, Ledesma MG, van Hmert J, Radwan A, Cavallerano JD, et al. Diabetic retinopathy severity and peripheral lesions are associated with no perfusion on ultrawide field angiography. Ophthalmology. 2015;122(12):2465-72.

6. American Academy of Ophthalmology. Basic and Clinical Science Course. Section 12. Retina and Vitreous. San Francisco, American Academy of Ophthalmology; 2012

7. Rajalakshmi R, Arulmalar S, Usha M, Prathiba V, Kareemuddin KS, Anjana $\mathrm{RM}$, et al. Validation of smartphone based retinal photography for diabetic retinopathy screening. PLoS One. 2015;10(9):e0138285.

8. Valverde C, García M, Hornero R, López-Gálvez MI. Automated detection of diabetic retinopathy in retinal images. Indian J Opthalmol. 2016:64(1):26-32.

9. Nagiel A, Lalane RA, Sadda SR, Schwartz SD. Ultra-widefield fundus imaging. A review of clinical applications and future trends. Retina. 2016; 36(4):660-78

10. Silva PS, Horton MB, Clary D, Lewis DG, Sun JK, Cavallerano JD. Identification of diabetic retinopathy and ungradable image rate with ultrawide field imaging in a national teleophtalmology program. Ophthalmology. 2016:123(6):1360-7.

11. Varner P. How frequently should asymptomatic patients be dilated? J Optom. 2014;7(1):57-61.

12. Jiménez-Báez MV, Márquez-González H, Bárcenas-Contreras R, MoralesMontoya C, Espinosa-García LF. Early diagnosis of diabetic retinopathy in primary care. Colomb Méd. 2015;46(1):14-8.

13. Graue-Hernández EO, Rivera-De-La-Parra D, Hernández-Jiménez S, AguilarSalinas CA, Kershenobich-Stalnikowitz D, Jimenez-Corona A. Prevalence and associated risk factors of diabetic retinopathy and macular oedema in patients recently diagnosed with type 2 diabetes. BMJ Open Ophthalmol. 2020:5:e000304.

14. Cockburn N, Steven D, Lecuona K, Joubert F, Rogers G, Cook C, et al. Prevalence, causes and socio-economic determinants of vision loss in Cape Town, South Africa. PLoS One. 2012;7:e30718.

15. Owsley C, McGwin G, Lee DJ, Lam BL, Friedman DS, Gower EW, et al. Diabetes eye screening in urban settings serving minority populations: detection of diabetic retinopathy and other ocular findings using telemedicine. JAMA Ophthalmol. 2015:133(2):174-81.

16. Scanlon PH, Aldington SJ, Stratton IM. Short report: Complications delay in diabetic retinopathy screening increases the rate of detection of referable diabetic retinopathy. Diabet Med. 2014;31:439-42.

17. Forster AS, Forbes A, Dodhia H, Connor C, du Chemin A, Sivaprasad S, et al. Changes in detection of retinopathy in type 2 diabetes in the first 4 years of a population-based diabetic eye screening program. Diabetes Care. 2013:36:2663-9.

18. Burgess PI, Msukwa G, Beare NAV. Diabetic retinopathy in Sub-Saharan Africa: meeting the challenges of an emerging epidemic. BMC Medicine. 2013;11:157. 